\title{
Efficient Data Mapping and Buffering Techniques for Multilevel Cell Phase-Change Memories
}

\author{
HANBIN YOON ${ }^{*}$ and JUSTIN MEZA, Carnegie Mellon University \\ NAVEEN MURALIMANOHAR, Hewlett-Packard Labs \\ NORMAN P. JOUPPI**, Google Inc. \\ ONUR MUTLU, Carnegie Mellon University
}

New phase-change memory (PCM) devices have low-access latencies (like DRAM) and high capacities (i.e., low cost per bit, like Flash). In addition to being able to scale to smaller cell sizes than DRAM, a PCM cell can also store multiple bits per cell (referred to as multilevel cell, or MLC), enabling even greater capacity per bit. However, reading and writing the different bits of data from and to an MLC PCM cell requires different amounts of time: one bit is read or written first, followed by another. Due to this asymmetric access process, the bits in an MLC PCM cell have different access latency and energy depending on which bit in the cell is being read or written.

We leverage this observation to design a new way to store and buffer data in MLC PCM devices. While traditional devices couple the bits in each cell next to one another in the address space, our key idea is to logically decouple the bits in each cell into two separate regions depending on their read/write characteristics: fast-read/slow-write bits and slow-read/fast-write bits. We propose a low-overhead hardware/software technique to predict and map data that would benefit from being in each region at runtime. In addition, we show how MLC bit decoupling provides more flexibility in the way data is buffered in the device, enabling more efficient use of existing device buffer space.

Our evaluations for a multicore system show that MLC bit decoupling improves system performance by $19.2 \%$, memory energy efficiency by $14.4 \%$, and thread fairness by $19.3 \%$ over a state-of-the-art MLC PCM system that couples the bits in its cells. We show that our results are consistent across a variety of workloads and system configurations.

Categories and Subject Descriptors: B.3.1 [Memory Structures]: Semiconductor Memories; C.0 [Computer Systems Organization]: General

General Terms: Design, Algorithms, Performance

Additional Key Words and Phrases: Multilevel cell, phase-change memory, main memory, performance, energy, data mapping, data buffering

\section{ACM Reference Format:}

HanBin Yoon, Justin Meza, Naveen Muralimanohar, Norman P. Jouppi, and Onur Mutlu. 2014. Efficient data mapping and buffering techniques for multilevel cell phase-change memories. ACM Trans. Architec. Code Optim. 11, 4, Article 40 (December 2014), 25 pages.

DOI: http://dx.doi.org/10.1145/2669365

\footnotetext{
*H. Yoon is currently at Google Inc.

*** This work was performed while the author was at Hewlett-Packard Labs.

This research was supported by NSF Awards 0953246, 1147397, 1212962, 1320531, the Intel Science and Technology Center for Cloud Computing, the Semiconductor Research Corporation, and the Intel Memory Hierarchy Program.

Authors' address: 5000 Forbes Ave, Pittsburgh, PA 15213; emails: hanbiny@alumni.cmu.edu, meza@cmu.edu, naveen.muralimanohar@hp.com, jouppi@google.com, onur@cmu.edu.

Permission to make digital or hard copies of part or all of this work for personal or classroom use is granted without fee provided that copies are not made or distributed for profit or commercial advantage and that copies show this notice on the first page or initial screen of a display along with the full citation. Copyrights for components of this work owned by others than ACM must be honored. Abstracting with credit is permitted. To copy otherwise, to republish, to post on servers, to redistribute to lists, or to use any component of this work in other works requires prior specific permission and/or a fee. Permissions may be requested from Publications Dept., ACM, Inc., 2 Penn Plaza, Suite 701, New York, NY 10121-0701 USA, fax +1 (212) 869-0481, or permissions@acm.org.
}

(c) 2014 ACM 1544-3566/2014/12-ART40 $\$ 15.00$

DOI: http://dx.doi.org/10.1145/2669365 


\section{INTRODUCTION}

There is a growing demand in computing systems, from mobile phones to servers, for larger-capacity main memories at lower cost. Traditional main memories have been composed of dynamic random-access memory (DRAM), a technology that is predicted to be difficult to scale to smaller cell sizes without greatly increasing energy consumption [The International Technology Roadmap for Semiconductors 2010]. To help address these trends, recent research has focused on the design and application of more scalable resistive memories [Raoux et al. 2008; Wong et al. 2010; Hosomi et al. 2005; Strukov et al. 2008]. In this work, we focus on one type of resistive memory, phase change memory (PCM), due to its potential to provide higher device capacity compared to DRAM [Lee et al. 2009, 2010b] and its ability to store multiple bits per cell (called multilevel cell, or MLC), which DRAM cannot. ${ }^{1}$

Prior work on PCM has focused primarily on device architectures and techniques to enable improved endurance, reduced access latency, and lower write latency/energy, which are agnostic to the number of bits per cell [Zhou et al. 2009; Qureshi et al. 2009a; Cho and Lee 2009; Ipek et al. 2010; Schechter et al. 2010; Yoon et al. 2011; Qureshi et al. 2009b; Lee et al. 2009; Qureshi et al. 2012; Lee et al. 2010a; Yoon et al. 2012; Meza et al. 2012a]. While such techniques can be applied to both single- or multilevel cell PCM, they do not take advantage of two of the unique aspects of MLC PCM. First, MLC PCM cell data is written iteratively: one bit is written first, followed by another [Bedeschi et al. 2009; Nirschl et al. 2007]. Second, MLC PCM cell data is read sequentially: the value of one bit can be detected before another [Qureshi et al. 2010b].

Such characteristics create asymmetry in the latencies and energies required for read and write operations in MLC PCM-varying by $16 \%$ to $80 \%$ depending on the before and after states of the cell [Bedeschi et al. 2009; Nirschl et al. 2007; Joshi et al. 2011; Qureshi et al. 2010b]. Keeping cell access latency low for MLC PCM cells is especially important because it can be a critical bottleneck in accessing device data, accounting for $99 \%$ of the overall device write latency and more than $95 \%$ of the overall device read latency [Qureshi et al. 2010a, 2010b; Lee et al. 2009], compared to other sources of delay such as the address and data wire routing (in contrast to devices like DRAM, in which cell access latency is small compared to address and data wire routing delays).

In this article, we leverage this asymmetry in the latencies and energies required for read and write operations in MLC PCM to design a new way to store and buffer data in MCL PCM devices. Our key idea is to logically decouple the bits in each cell into two separate regions depending on their read/write characteristics: fast-read/slow-write (FR) bits and slow-read/fast-write (FW) bits. ${ }^{2}$ Based on this key idea, our technique, called decoupled bit mapping (DBM), exposes to the system two logical regions in an MLC PCM device (using one control bit to differentiate between the two): (1) a region with low latency and low energy for reads (which uses the FR bits), and (2) a region with low latency and low energy for writes (which uses the FW bits).

We also propose a new low-overhead hardware/software technique to take advantage of a DBM-enabled MLC PCM device to make better page placement decisions (similar to the goal of Ramos et al. [2011], Yoon et al. [2012], and Dhiman et al. [2009]). Our asymmetric page mapping $(A P M)$ technique uses a small set of hardware counters to track the read/write intensity of the most active load and store instructions in a program. When the operating system (OS) is about to allocate a new physical page in MLC PCM, it queries a hardware structure with the address of the load or store

\footnotetext{
${ }^{1}$ Though we perform our quantitative evaluations using MLC PCM as an example device, our techniques are applicable to other resistive memories with similar characteristics to MLC PCM.

${ }^{2}$ Note that, concurrent to our work [Yoon et al. 2013], Jiang et al. [2012a] made a similar observation in the context of STT-RAM caches. In that article, FR bits are called RFWS bits and FW bits are called RSWF bits.
} 
instruction that initiated the page allocation, and receives a prediction of whether the page will be (1) read-intensive or (2) write-intensive. Pages predicted to be readintensive are mapped to the FR region of a DBM-enabled MLC PCM device, while pages predicted to be write-intensive are mapped to a device's FW region.

Finally, we design a new technique that shows how the MLC bit decoupling enabled by DBM provides more flexibility in the way data is buffered in an MLC PCM device. Internally, memory devices buffer a small amount of data being read or written in a structure called the row buffer. Modern MLC PCM devices with coupled bit mappings utilize a row buffer organization that joins together the bits of a cell in the row buffer. We show that by logically decoupling the bits of a cell with DBM, using the same row buffer space and only a small amount of additional control circuitry, we can split the row buffer space between two logically separate row buffers: a row buffer for FR bits and a row buffer for FW bits. We show that our split row buffer (SRB) organization enables more efficient use of existing device buffer space, similar to if row buffer space had been doubled, but without the large overhead.

We make the following contributions:

-A technique developed concurrently with Jiang et al. [2012a] for mapping the bits in MLC PCM cells that exposes to the system two logical regions: (1) a region with low latency and low energy for reads and (2) a region with low latency and low energy for writes ${ }^{3}$. Unlike Jiang et al., who examined the applicability of their technique in the context of STT-RAM caches, we leverage and evaluate our technique in the context of a PCM main memory.

-A hardware/software technique to predict and map data that would benefit from being in each DBM region at runtime based on its read/write characteristics. To make the prediction, we use a low-overhead hardware table to track the read/write characteristics of a small number of the most active load and store instructions.

-A new row buffer organization for MLC PCM devices, enabled by DBM, that provides logically separate row buffers for each of the different bits in an MLC, with only a small amount of additional control circuitry. We show that our SRB organization enables more efficient use of existing device buffer space.

-An evaluation of our schemes for a multicore system showing that the combination of our techniques improves system performance by $19.2 \%$, memory energy efficiency by $14.4 \%$, and thread fairness by $19.3 \%$ over a state-of-the-art MLC PCM system that couples the bits in its cells. We show that our results are consistent across a variety of workloads and system configurations.

\section{BACKGROUND}

\subsection{Memory Device Architecture}

Memory devices consist of arrays of cells and peripheral circuitry. Figure 1 shows a memory device array, where cells are organized into rows and columns. All of the cells in a row are connected to a common word line, and all of the cells in a column are connected to a common bit line. To simplify the device organization, when accessing data in the array, an entire row of cells is accessed simultaneously. To do this, a row decoder asserts a word line to select all of the cells in a target row, and the bit lines transmit data between the cells and peripheral circuits.

In the peripheral circuits, data signals from the bit lines are detected by sense amplifiers and latched in the row buffer, and a column decoder selects a subset of the row buffer to be transmitted to the I/O pads. Once a row's data is placed in the row

${ }^{3}$ Though we focus on MLC PCM devices with two bits per cell, our technique can be generalized to support devices with more bits per cell. 


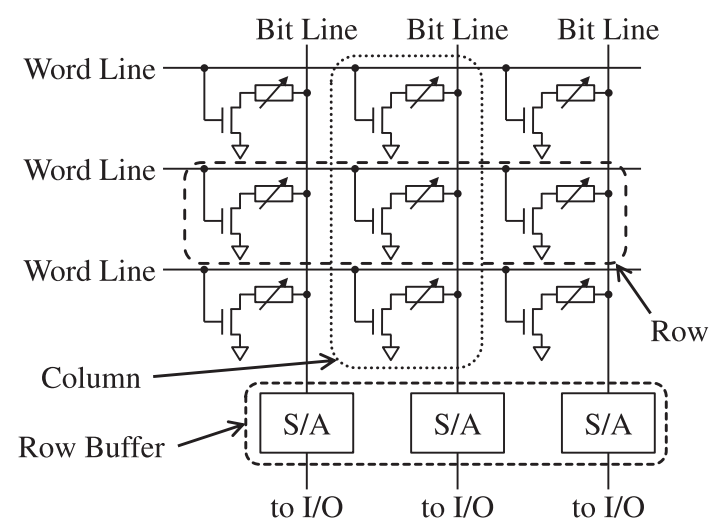

Fig. 1. Memory cell array shown with PCM cells. S/A = sense amplifier.

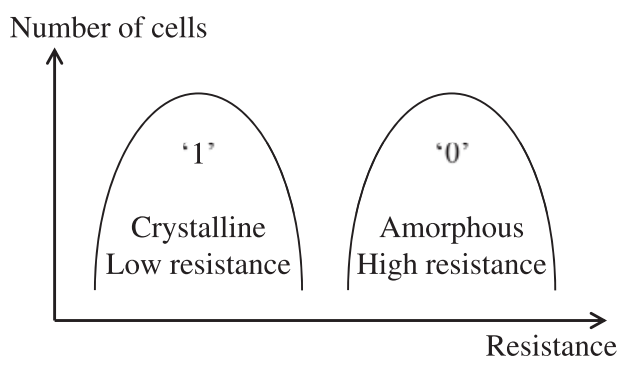

(a) Single-level cell resistance distribution.

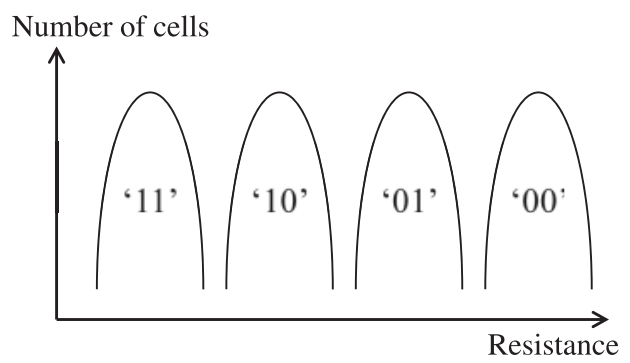

(b) Multi-level cell resistance distribution.

Fig. 2. PCM cell resistance distribution.

buffer, subsequent data requests to the same row can be served by accessing the data in this buffer. Such an access is known as a row buffer hit, and can be served quickly at the access latency of the row buffer without interacting with the slower cell array. In order to serve a data request to a different row, data must be accessed from the array (replacing the contents of the row buffer). This type of access is known as a row buffer miss, and incurs higher latency and higher energy consumption than a row buffer hit because it must perform the previously described steps to access a row of cells in the array.

\subsection{Phase Change Memory}

Phase-change memory (PCM) is a memory technology that stores data in a cell by varying the electrical resistance of a material called chalcogenide [Raoux et al. 2008; Wong et al. 2010]. By applying heat and then allowing it to cool at different rates, chalcogenide can be manipulated to settle between an amorphous (quickly cooled) high-resistance state, and a crystalline (slowly cooled) low-resistance state. Each state corresponds to a nonoverlapping resistance range, and the resistance of cells programmed to the same value falls into the same range, but their exact resistance could be different (due to process variation). For example, Figure 2(a) illustrates the distribution of cells with different resistance values if all cells were programmed with the value 0 or the value 1 . The nonoverlap space between the ranges is called the distribution margin. Because the physical state of each cell is modified when it is programmed, PCM is able to retain the data stored in its cells in the absence of power, making it a nonvolatile memory technology. 
Compared to DRAM cells, which store data using electrical charges in a capacitor, PCM cells have been shown to be easier to shrink to smaller sizes [Wong et al. 2010]. This gives PCM devices the potential to provide higher density, lower cost per bit, and larger capacity than DRAM [Lee et al. 2009]. In addition, PCM's low static power further adds to its competitiveness as a scalable DRAM alternative.

However, PCM exhibits several drawbacks compared to DRAM, as described by Lee et al. [2009]. Cell access latency may be 4 to $32 \times$ larger depending on the operation and cell access energy is around $2 \times$ for reads and 10 to $140 \times$ for writes compared to DRAM. PCM cells also may only be written around $10^{6}$ to $>10^{9}$ times, compared to DRAM cells, which may be written an estimated $10^{17}$ times [The International Technology Roadmap for Semiconductors 2010].

PCM devices are still in an early phase of development and deployment, and many solutions have been proposed to overcome PCM's shortcomings. Some proposals include DRAM caching [Qureshi et al. 2009b], fine-grained buffering of row data [Lee et al. 2009], wear-leveling/-reduction [Qureshi et al. 2009a; Cho and Lee 2009; Zhou et al. 2009], and fault-tolerance techniques [Ipek et al. 2010; Schechter et al. 2010; Yoon et al. 2011].

\subsection{Multilevel Cell Memory}

Multilevel cell (MLC, or multibit cell) refers to a memory cell's ability to store more than one bit of data. Multiple bits can be stored in a PCM cell by programming and sensing additional resistance states. Figure 2(b) shows how two additional resistance states could be added to a cell to enable it to encode two bits of data. As a result, MLC devices can store more data per unit area compared to cells with only a single level.

Notice that compared to Figure 2(a), the range of resistances that correspond to each state is smaller, which requires more accurate programming and sensing. Due to this, MLC cells are read and written using techniques that have higher access latency and higher access energy compared to cells with only a single level [Bedeschi et al. 2009; Nirschl et al. 2007; Qureshi et al. 2010b].

One challenge in the adoption of MLC memory devices is the phenomenon of resistance drift, which has been documented in PCM devices [Seong et al. 2013]; a similar issue has been identified in other MLC nonvolatile memories such as Flash [Cai et al. 2012, 2013]. Resistance drift occurs when the resistance of a cell changes over time due to disturbance caused by accessing nearby cells in a device or due to changes in the cell material structure due to repeated accesses over time. Recent work [Seong et al. 2013] has examined ways to mitigate this effect by using the appropriate number of resistance ranges for MLCs. We believe that such techniques can be used to help mitigate the effects of resistance drift in MLC PCM devices and enable their reliable use.

\section{MOTIVATION}

\subsection{Cell Read/Write Asymmetries in MLC PCM}

Due to the way that data is read from and written to MLC PCM cells, each bit in an MLC cell requires a different amount of latency and energy to read or write. We next describe why these asymmetries occur and how we can leverage them to design more efficient techniques to manage MLC PCM devices.

3.1.1. Cell Read Asymmetry. The read latency of an MLC PCM cell depends on the state of the cell. This is illustrated in Figure 3(a), which depicts an MLC PCM read operation on a cell with four levels (corresponding to two bits of stored data). An integrating analog-to-digital converter quantizes the resistance of an MLC PCM cell to a two-bit value (we refer to the left bit as the most significant bit, MSB, and the right bit as the 


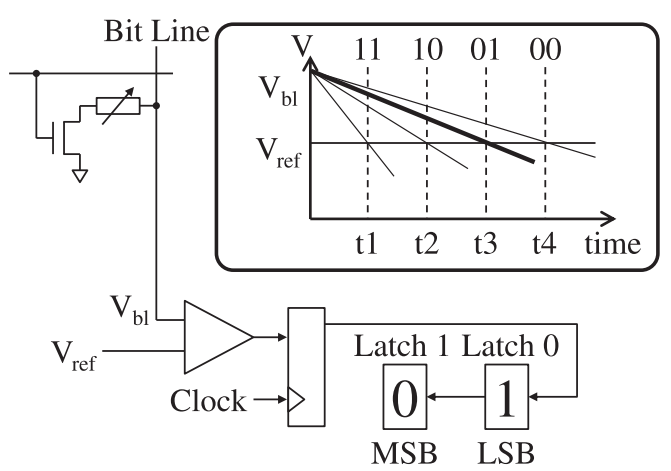

(a) Sensing time is longer for higher cell resistances.

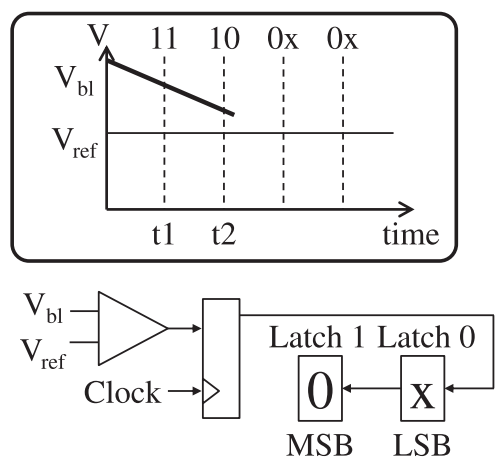

(b) One bit is determined before the other.

Fig. 3. MLC PCM cell read operation [Qureshi et al. 2010b].

least significant bit, LSB) by measuring the time taken to send an electrical current from the bit line through the cell. A cell in a lower resistance state will take less time to propagate the current than a cell in a higher resistance state. Therefore, the contents of a cell can be determined by monitoring the time it takes for the bit line voltage $\left(\mathrm{V}_{\mathrm{bl}}\right.$ in Figure 3(a)) to reach a reference voltage $\left(\mathrm{V}_{\text {ref }}\right)$. In Figure 3(a), it took t3 amount of time for $V_{b l}$ to reach $V_{\text {ref }}$, thus the corresponding value of the MSB is 0 and the LSB is 1 .

However, notice that some information is available about the contents of the cell even before time t3. As Figure 3(b) shows, after time t2, we may eliminate the possibility of the cell contents being either 11 or 10 , because if it contained either of these values, $\mathrm{V}_{\mathrm{bl}}$ would have to be $\leq \mathrm{V}_{\text {ref }}$ by time $\mathrm{t} 2$, which it is not. Since the only remaining values that the cell may contain are 01 or 00 , after time t2, it must be the case that the MSB (the left bit) is 0 . Thus, due to cell read asymmetry, one of the bits in an MLC PCM cell (the MSB bit in our example) can be accessed with lower read latency and lower read energy than the other.

This property of MLC PCM cells is not exploited in conventional architectures, however, because all of the bits in each cell belong to the same block of data, thus must always be accessed together. In other words, in addition to being physically coupled together, the bits in MLC PCM cells in traditional architectures are also logically coupled together. By decoupling the bits in an MLC PCM cell, we can more efficiently read data from one of the bits in the cell.

3.1.2. Cell Write Asymmetry. The write latency and write energy of an MLC PCM cell depend on (1) the initial state of the cell and (2) the final state of the cell. This is because transitioning a cell between the different resistance states in Figure 2(b) requires different amounts of power to be applied over different amounts of time. There are two techniques for programming PCM cells: (1) partially crystallizing amorphous chalcogenide and (2) partially amorphizing crystalline chalcogenide. MLC PCM write operation is optimized to use the faster of these two techniques for each transition [Nirschl et al. 2007; Happ et al. 2006; Joshi et al. 2011] provide more detail on the implementation and application of these techniques. ${ }^{4}$

Figure 4(a) shows the amount of time taken to transition a two-bit MLC PCM cell between any two states (normalized to the slowest transition latency). There are two

\footnotetext{
${ }^{4}$ While it remains to be seen whether the programming techniques developed in these works will eventually become the dominant type of programming scheme for MLC PCM cells, we note that other nonvolatile storage technologies such as Flash have successfully adopted similar techniques (e.g., Suh et al. [1995]), and we believe that nonvolatile memory devices will be able to do so as well.
} 


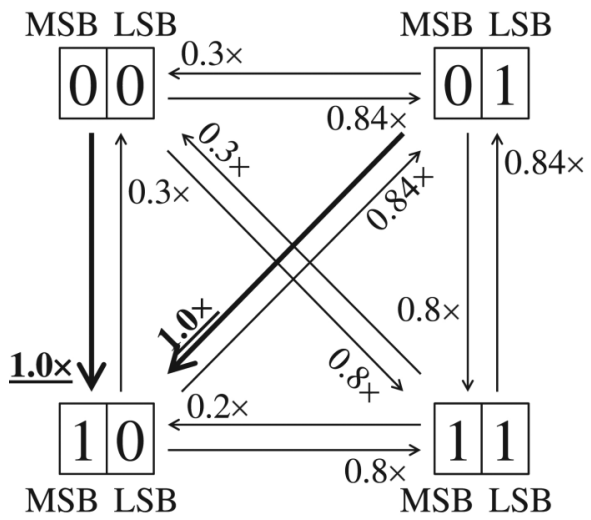

(a) All possible cell state transitions.

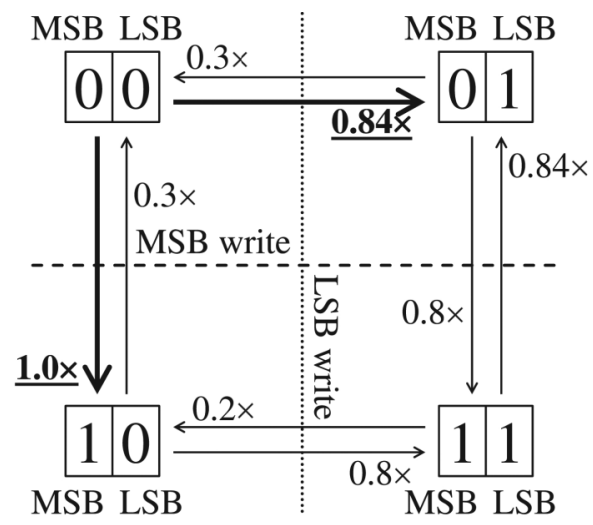

(b) Cell state transitions when modifying only the MSB or the LSB.

Fig. 4. MLC PCM cell write latencies [Joshi et al. 2011; Nirschl et al. 2007; Happ et al. 2006].

things to note in Figure 4(a): (1) the worst-case latencies among all the possible transitions correspond to changing the value of the MSB (from 0 to 1 , shown by the bold lines) and (2) the worst-case latency among all the possible transitions for changing the LSB is only $0.84 \times$ (corresponding to changing the LSB from 0 to 1 ). This suggests that if we could isolate which of the bits in an MLC PCM cell we change at one time, we could guarantee lower write latency for LSB bit changes. Figure 4(b) shows the normalized state transition latency when a cell may change only its MSB (corresponding to vertical transitions) or its LSB (corresponding to horizontal transitions) but not both in the same write operation. Applying this constraint shows that while the worst-case write latency for the MSB is $1.0 \times$, the worst-case write latency for the LSB is bounded at $0.84 \times$. Thus, due to cell write asymmetry, one of the bits in an MLC PCM cell (the LSB bit in our example) can be written with lower write latency and lower write energy than the other.

Similar to cell read asymmetry, this property of MLC PCM cells is also not exploited in conventional architectures because all of the bits in a cell belong to the same block of data and must therefore always be modified together. By logically decoupling the bits in an MLC PCM cell, we can more efficiently write data to one of the bits in the cell.

3.1.3. Key Observation. Notice from our discussion that the bit that provides the best read latency and read energy (the MSB bit in our examples) is different from the bit that provides the best write latency and write energy (the LSB bit in our examples). Our key observation is that the bits in an MLC PCM cell can be used in a way that complement each other in terms of their read/write characteristics - a property that we propose to leverage. We hereafter refer to bits that provide the best read latency and read energy as fast-read/slow-write $(F R)$ bits, and bits that provide the best write latency and write energy as slow-read/fast-write $(F W)$ bits.

\subsection{Leveraging Read/Write Asymmetry}

We leverage MLC PCM cell read/write asymmetry to logically decouple the bits of each cell to create two regions of memory: (1) a region composed of FR bits that is more efficient for reading data and (2) a region composed of $\mathrm{FW}$ bits that is more efficient for writing data. We call this technique decoupled bit mapping, or DBM. Note that a similar mechanism was concurrently proposed for STT-RAM caches [Jiang et al. 2012a]. The main contribution with respect to our technique is its evaluation in the context of 


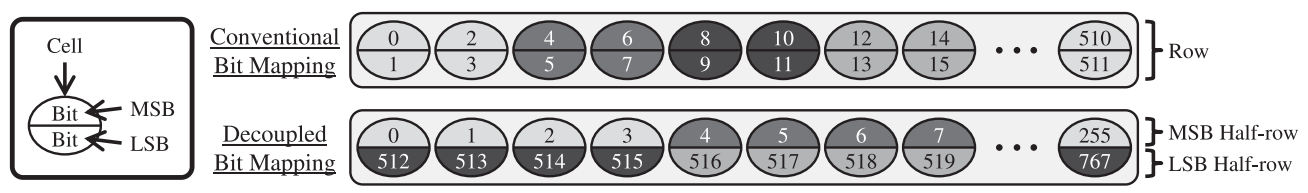

Fig. 5. Different MLC PCM bit mapping schemes that we examine. (Note that we only show the first 256 cells in a row.)

and MLC PCM main memory, and the proposal and evaluation of two new techniques that leverage it to achieve higher performance and higher energy efficiency. We next provide an overview of these techniques and describe their detailed implementations in Section 5. An evaluation is presented in Section 7.

Figure 5 illustrates the difference between a conventional bit mapping scheme and a decoupled bit mapping scheme. In this figure, MLC PCM cells are represented as circles divided into each of their corresponding bits (with the MSB on the top and the LSB on the bottom). Cache blocks, whose bits are all read or written at the same time, are shown using different shades of gray (we assume, for illustrative purposes, that the size of a cache block is four bits in Figure 5). In Figure 5, 256 cells comprise a physical row, and one physical row of cells is shown for each of the mapping schemes.

In the conventional bit mapping scheme, cache blocks are formed using all of the bits in each of two adjacent MLC PCM cells. In this scheme, reading a cache block requires accessing all of the bits in two cells (incurring the longer latency of reading LSBs), and writing a cache block requires potentially modifying all of the bits in two cells (potentially incurring the higher latency and higher energy of modifying MSBs).

With a decoupled bit mapping scheme, a cache block is formed using either (1) the FR bits (located in the MSB half-row in Figure 5) in four adjacent MLC PCM cells or (2) the FW bits (located in the LSB half-row in Figure 5) in four adjacent MLC PCM cells. In this scheme, reading a cache block requires accessing only the FR (or FW) bits in four cells (with lower latency and lower energy for the cache blocks mapped to FR bits), and writing a cache block requires modifying only the FR (or FW) bits in four cells (with lower latency and lower energy for the cache blocks mapped to FW bits).

In this way, DBM provides a substrate for developing efficient MLC PCM techniques. As part of our research, we have identified two such techniques that can be enabled by DBM: (1) asymmetric page mapping (APM) and (2) split row buffering (SRB). Our first technique, APM, identifies data that has an affinity toward being read or written, and allocates that data in the appropriate DBM region. It does so using a small hardware table to predict the affinity of data toward being read or written and OS support to map pages of data to different regions of the physical address space, corresponding to the FR and FW regions of DBM. Our second technique, SRB, leverages the fact that a read or write operation in DBM manipulates either FR bits or FW bits but not both at the same time. Given this, with only a small hardware addition, the same row buffer area in MLC PCM devices can be divided into two separate row buffers, one for FR bits and other for FW bits, which can be manipulated individually to keep more data with locality in the row buffer.

The benefits of DBM, APM, and SRB are illustrated in Figure 6, which compares the memory service timelines between the conventional MLC PCM architecture (a) and systems employing these schemes (b-c), for an example memory access pattern. Compared to the conventional scheme, because of how data is statically mapped, DBM is able to serve read requests to blocks 17 and 18 at lower latency and lower energy, as these blocks now occupy only FR bits (additionally, blocks in the FW bits can be written to at lower latency and lower energy). With APM, the OS allocates block 1 to an FW region (whereas this data would have been allocated to the FR region using a 


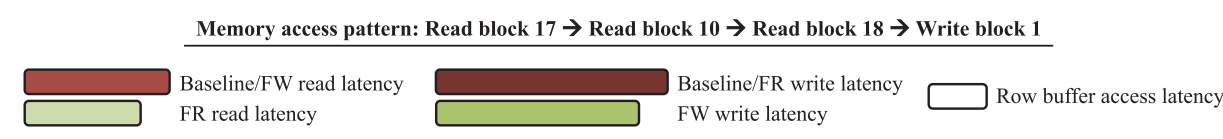

(a)
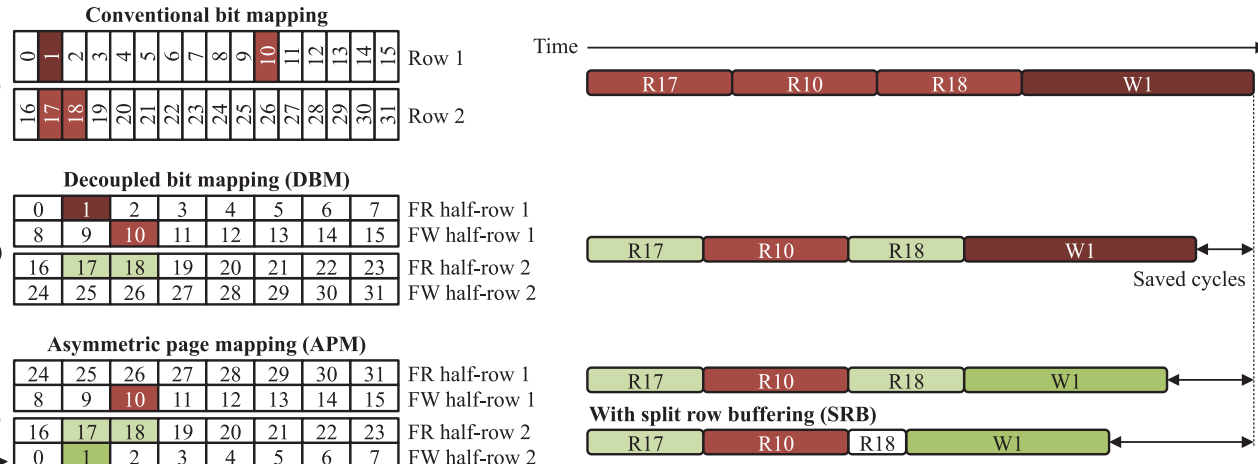

(c)

Decoupled bit mapping (DBM)

(b) \begin{tabular}{|c|c|c|c|c|c|c|c|c|}
\hline 0 & 1 & 2 & 3 & 4 & 5 & 6 & 7 & FR half-row 1 \\
\hline 8 & 9 & 10 & 11 & 12 & 13 & 14 & 15 & FW half-row 1 \\
\hline \hline 16 & 17 & 18 & 19 & 20 & 21 & 22 & 23 & FR half-row 2 \\
\hline 24 & 25 & 26 & 27 & 28 & 29 & 30 & 31 & FW half-row 2 \\
\hline
\end{tabular}

Asymmetric page mapping (APM)

\begin{tabular}{|c|c|c|c|c|c|c|c|c|}
\hline 24 & 25 & 26 & 27 & 28 & 29 & 30 & 31 & FR half-row 1 \\
\hline 8 & 9 & 10 & 11 & 12 & 13 & 14 & 15 & FW half-row 1 \\
\hline 16 & 17 & 18 & 19 & 20 & 21 & 22 & 23 & FR half-row 2 \\
\hline 0 & 1 & 2 & 3 & 4 & 5 & 6 & 7 & FW half-row 2 \\
\hline
\end{tabular}

Fig. 6. Decoupled bit mapping can improve performance compared to a conventional bit mapping, with the potential to further improve performance using asymmetric page mapping and split row buffering.

static mapping scheme). Thus, APM is able to exploit the lower write latency and write energy of FW bits to serve the write request to block 1. Serving the read request to block 18 results in a row buffer miss access without $\mathrm{SRB}$, as the row buffer will contain the row with the previously accessed block 10. However, with SRB, blocks 17 and 18 can simultaneously be buffered in the row buffer, causing the read request to block 18 (which is buffered along with block 17) to be a row buffer hit access. This further reduces the time and energy taken to serve the memory requests.

\section{DETAILED MECHANISMS}

\subsection{Decoupled Bit Mapping (DBM)}

DBM is designed to expose the lower latency and lower energy of FR bit reads and FW bit writes. To this end, the bits of an MLC cell are mapped to separate logical addresses, as illustrated in Figure 5. Whereas these bits are coupled to form a single contiguous memory address along a row in the conventional scheme, DBM groups the FR bits along a row to form one contiguous address, and groups the FW bits along the same row to form another. This way, a data block (e.g., a 64B cache block) that resides at a certain logical address physically occupies only FR bits or only FW bits. If the data block is in FR bits, read asymmetry is exploited to read the block at a reduced latency and energy (the FR region). If the data block is in FW bits, write asymmetry is exploited to write to the block at a reduced latency and energy (the FW region).

DBM divides all rows in memory (i.e., all cells that share a common word line) into two logical regions: one consisting of FR bits, the other consisting of FW bits. Assuming a uniformly random translation from an application's virtual page address to a physical page address in memory, approximately half of the application's working set would be in FR bits and the other half in FW bits. Hence with DBM, on average, $50 \%$ of the data in memory may be served at reduced read latency (by 48\%) and read energy (by 48\%), and $50 \%$ of the data in memory may be served at reduced write latency (by 16\%) and write energy (by 26\%).

The downside of DBM is that it may increase the average number of cells that are programmed during a write operation, potentially wearing out more cells more quickly. This is because, with DBM, a data block uses only one bit from each multibit cell, 
potentially involving a number of cells equal to the number of bits in a block when writing to the block. However, this does not double the write endurance overhead compared to the conventional scheme, as the probability of redundant bit-writes [Zhou et al. 2009] (where the cell is already in the target state to be programmed to, thus avoiding a programming operation) is lower in the conventional scheme. For programming a cell to be redundant in the conventional scheme, both the FR bit and the FW bit have to match with the write data. On the other hand, write data to a block in DBM targets only FR bits or only FW bits. If the write is to FR bits, the FW bits are unchanged, and vice versa. Because only one bit per cell needs to match with the write data in DBM, the probability of redundant bit-writes is higher in DBM than in the conventional scheme (also noted by Zhou et al. [2009] and Jiang et al. [2012b]), and we observe on average a $28.2 \%$ endurance overhead in our results (discussed in Section 7.4). As we will show, this is small enough to achieve a typical server operational lifetime of 5 years (server installations typically have a lifetime of 3 to 4 years [Barroso et al. 2013]), even assuming pessimistic PCM cell wearout characteristics.

By employing DBM, two separate logical address spaces share the row buffer space, with data from each address space occupying half of the row buffer. This reduces the longest contiguous address space that can be held in the row buffer, potentially decreasing row buffer locality. However, the reduced memory access latency and energy exposed by DBM have the potential to more than compensate for this effect, and significantly improve system performance and energy efficiency over the conventional MLC PCM architecture, while not incurring any major modifications to the memory circuitry (we detail our implementation in Section 5). To utilize read and write asymmetries more effectively, we next propose a technique to map a page to either the FR region or FW region depending on the page's memory read/write affinity.

\subsection{Asymmetric Page Mapping (APM)}

With DBM alone, it is not possible to ensure that a page will get mapped to the most beneficial region of memory in terms of reduced latency and energy. This is because only half of the memory space is efficient in serving reads (the FR region), while the other half of memory is efficient in serving writes (the FW region), yet OS physical page allocation may distribute pages arbitrarily between both regions (an effect we model in our evaluations). However, many applications exhibit skewed page-access behavior where a small fraction of pages account for a large portion of the memory accesses. For example, Table I shows this trend for SPEC CPU2006 benchmarks, listing the fraction of pages that account for $80 \%$ of the memory reads and $80 \%$ of the memory writes in the second and third columns ${ }^{5}$. On average, $80 \%$ of the reads and writes are accounted for by $41 \%$ and $22 \%$ of the pages, respectively.

To understand how reads and writes are distributed within pages, we examined the ratio of reads to writes (and writes to reads) for frequently accessed pages that receive $90 \%$ or more of the reads or writes in the program. The fourth column shows the fraction of frequently read pages (those that service $>90 \%$ of reads in the program) with a read-to-write ratio greater than 10 . These pages are both frequently read and read-intensive. The fifth column shows the fraction of frequently written pages (those that service $>90 \%$ of writes in the program) with a write-to-read ratio greater than 2 (in general, the programs we examine are more read intensive than write intensive, so we adjust our standard for write intensity accordingly). These pages are both frequently written and write-intensive. This fraction is greater than $80 \%$ for 17 benchmarks in either reads or writes, indicating that many applications frequently access pages with

\footnotetext{
${ }^{5}$ In our evaluation, we examine workloads consisting of a subset of these benchmarks in addition to other parallel and multithreaded workloads (see Section 6).
} 


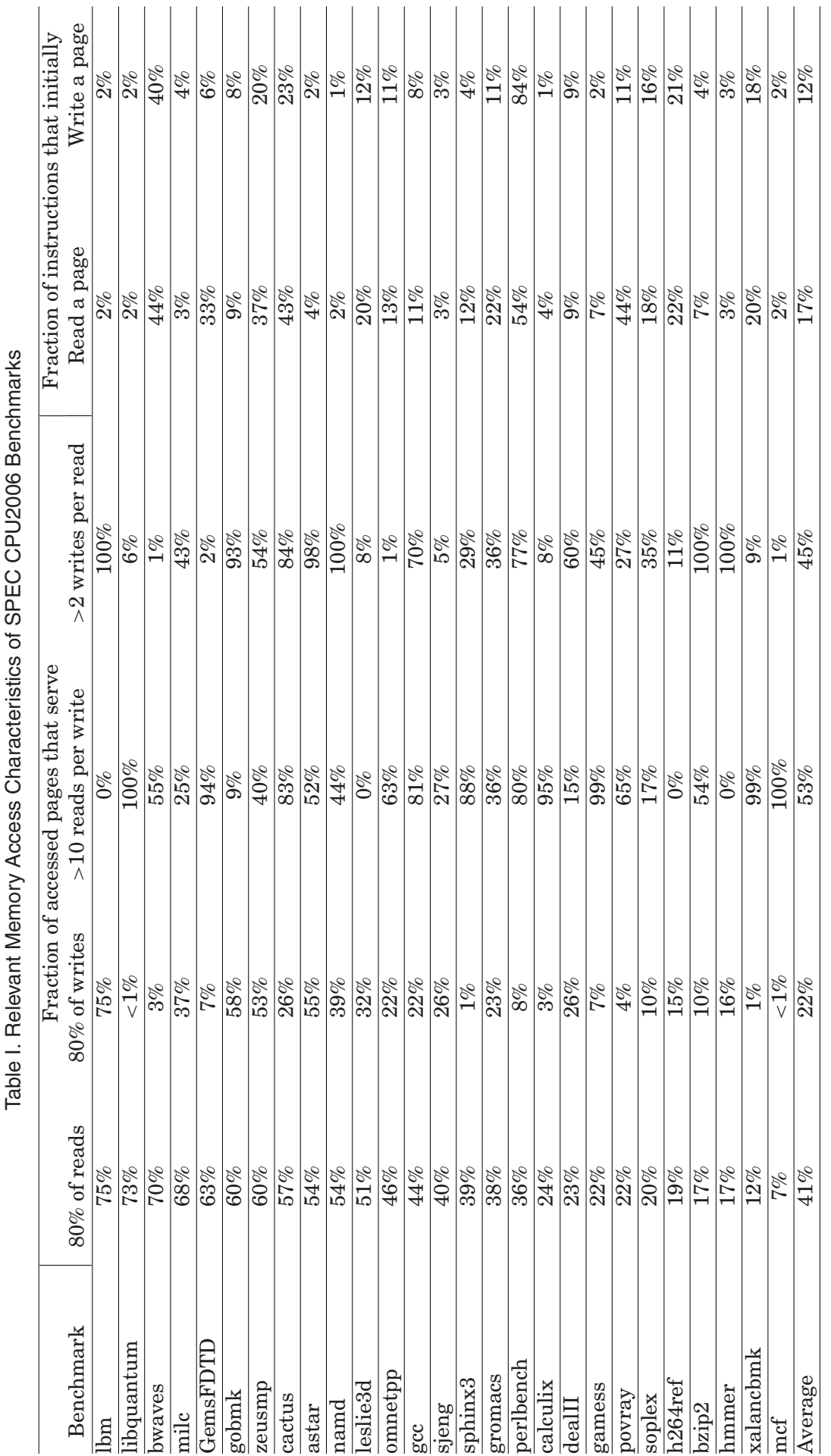


a strong bias toward reads or writes. Thus, by identifying and placing these accessintensive pages in memory regions that can efficiently serve the type of access (read or write) the pages frequently receive, it is possible to serve a larger portion of memory requests at reduced latency and energy.

There are two main approaches to achieving this page placement: reactive migration of a page to the correct region of memory and proactive allocation of a page to the correct region of memory. The first approach is to monitor the memory access patterns to pages, and migrate access-intensive pages to favorable regions (read-intensive pages to the FR region and write-intensive pages to the FW region) if they are not already placed in such regions by a default mapping. This straightforward approach allows matching pages to regions with high confidence, based on the observed memory access characteristics of pages. However, the two main disadvantages of this approach are that it (1) incurs high endurance and energy overhead, and (2) increases bank occupancy due to the movement of data in memory. An alternative approach is to predict the memory access patterns of pages and place them in favorable regions at their initial allocation. This method avoids the shortcomings of the first approach; however, it requires accurate predictions of the memory read/write characteristics of pages to be allocated.

We devise asymmetric page mapping (APM), which makes this prediction and advises the OS page allocation routine on the type of region (FR or FW) to map the page to. APM predicts whether the page to be allocated will be read-intensive or writeintensive, ${ }^{6}$ which the OS allocates to physical pages in FR or FW regions, respectively. If a physical page in the predicted region is unavailable, the page is allocated to the other region.

A key concern for our mechanism is how to efficiently predict where to map pages between the FR and FW regions. In our examination from Table I, we found that typically fewer instructions in a program initially access (and trigger the OS to allocate physical pages for) pages that experience the majority of memory writes (the sixth column, $17 \%$ on average), compared to the case for reads (the seventh column, $12 \%$ on average). ${ }^{7}$ We leverage this asymmetry in program access characteristics to track the smaller set of instructions that initially write pages (as opposed to read pages) to determine which of those instructions' allocated pages we predict should be placed in the FW region.

Leveraging this, APM uses a table to track the $N$ instructions (identified by the program counter) that generate the most writes to memory, and predicts a page to be write-intensive if its first-touch instruction is one of those $N$ instructions; otherwise, the page is predicted to be read-intensive. We find that using $N=16$ achieves the system performance within $1 \%$ of using $N=256$, so we adopt $N=16$ for our study.

To approximate the 16 instructions at run-time, a hardware program counter (PC) table of 16 entries is maintained in the processor core, as shown in gray in Figure 7. Each entry in the PC table holds an instruction address and a corresponding counter for counting the number of memory writes (dirty evictions from the cache) caused by the instruction. The PC table is used to identify the instructions with the number of memory writes they generated, while evicting instructions with low memory write counts from the PC table. In order to track the instruction that wrote to a cache block, each tag entry in the cache is augmented with a four-bit field (also gray in Figure 7)

\footnotetext{
${ }^{6}$ Although writes are not on the critical path, they occupy the banks for a long time $(8 \times$ that of reads); reducing this latency decreases the queuing delay of read requests.

${ }^{7}$ Modern operating systems typically allocate a new physical page for a virtual page only when the data is accessed in the memory for the first time, as opposed to when it is allocated [Kaminski 2009; Buros 2012; Doerner 2012].
} 


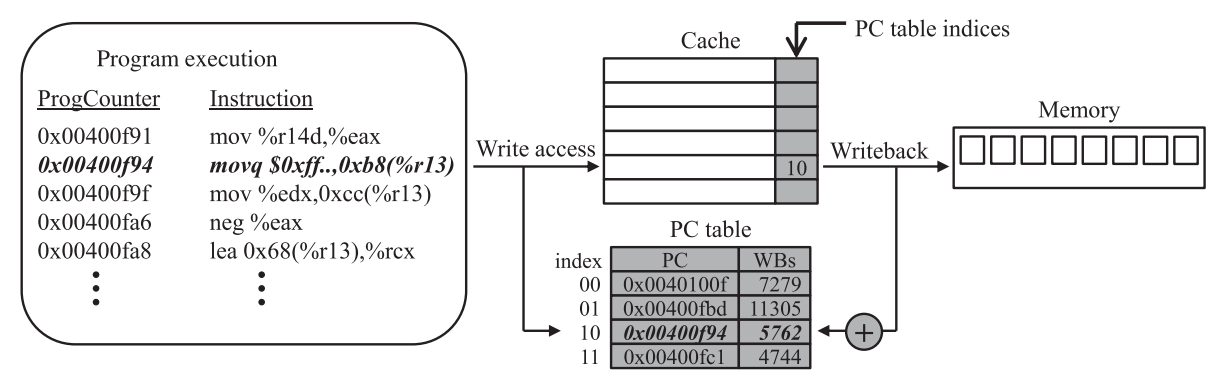

Fig. 7. PC table that tracks the instructions that generate the most writes to memory.

that stores an index value to the PC table that contains the instruction that wrote to the cache block (which we call the first-touch instruction).

The PC table operates as follows: For every write to the cache during the execution of a program, the address of the instruction (in the program counter) causing the write is associatively checked to see if it matches with any of the instruction addresses in the PC table. If there is no match, the instruction in the PC table with the smallest memory-write counter value is replaced with the instruction address of the write access, and the corresponding counter value is reset to 0 . Now that there is an entry in the $\mathrm{PC}$ table with the instruction that caused the write access, the index of this entry is stored in the tag store, in the four-bit field associated with the cache block that was written to. In the event that this cache block is written back to memory, its associated index value is used to index the PC table and increment the memory-write counter. Because entries in the PC table may be replaced with new instructions, it is possible for a memory-write counter to be incremented for an instruction that has already been evicted from the PC table.

During OS page allocation, a custom instruction is executed that checks whether the page's first-touch instruction address matches with any of the instruction addresses in the PC table. If there is a match, a register flag is set signalling that the page is predicted to be write-intensive (otherwise the page is predicted to be read-intensive). Depending on the prediction, the OS allocates the page contents to either a page in the FR region or a page in the FW region (which can be differentiated by a single regionselect bit in their address, as we describe in Section 5). All memory-write counters in the PC table are quartered (by right-shifting them twice) every 10 million cycles, which we empirically determined to take into account enough hysteresis information to adjust for phase changes in the program.

\subsection{Split Row Buffering (SRB)}

So far, we discussed how DBM exploits read/write asymmetry in MLC PCM to provide reduced memory access latency and energy, and how APM effectively utilizes DBM to make better data placement decisions. In addition to these benefits, DBM enables more flexibility in managing the row buffer, which is not possible in conventional MLC architectures.

In multilevel PCM, a sensing circuit for a bitline (recall Figure 3(a)) should have as many latches as the number of bits stored in a cell to be able to retrieve all of the bits of a cell. This is true for both the conventional MLC PCM architecture and our proposal, since sensing the MSB requires the LSB value to be first stored as shown in Figure 3.

DBM logically splits a row into two halves, each with their own contiguous logical addresses. This enables the row buffer to be manipulated as two half-row buffers, as shown in Figure 8. One of the two latches in every sensing circuit along a row buffer is grouped to form one half-row buffer; the other latches along the same row buffer are 


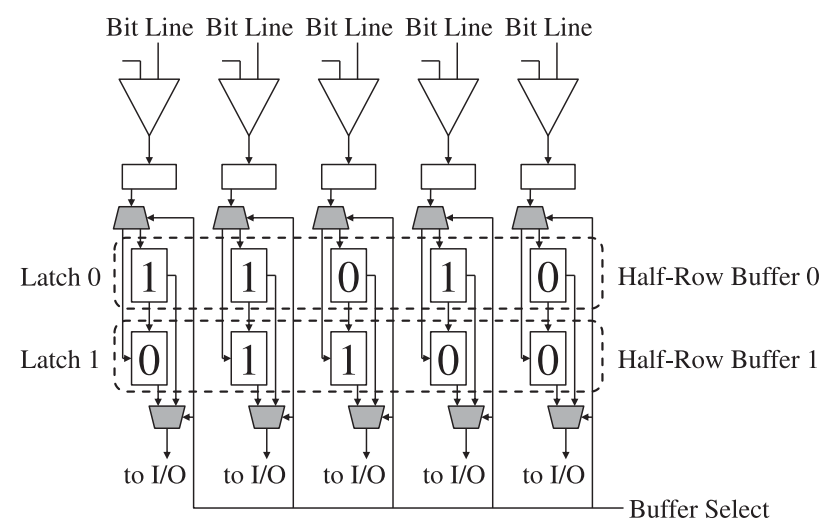

Fig. 8. With split row buffering, the row buffer is manipulated as two half-row buffers, storing FR bits or FW bits in each half.

grouped to form another half-row buffer. We propose a fine-granularity organization of half-row buffers that we call split row buffering (SRB).

SRB enables one half-row buffer to store FR bits from one set of contiguous addresses and another half-row buffer to store FW bits from another set of contiguous addresses. The two half-row buffers are used associatively: On a row buffer miss access, the least recently used half-row buffer's content (out of the two half rows in the full row) is chosen for eviction. SRB has an effect similar to adding another row buffer to the memory device, but at lower hardware cost (we provide implementation details in Section 5). This half-row buffer organization can increase the row buffer hit rate in MLC memory compared to when using a single full-row buffer, as we will show, thereby achieving higher performance and higher energy efficiency.

A subtle limitation of SRB is that while two FR half-rows can be simultaneously buffered, two FW half-rows cannot. This is because, as we showed in Section 3.1, though sensing an FR bit can be performed using a single latch in the sensing circuit, sensing an FW bit needs both latches as, without adding additional buffering circuitry, it requires the additional latch when reading the LSB (see Figure 3). Hence, reading FR bits into a half-row buffer can be done without affecting the contents of the other half-row buffer, whereas reading $\mathrm{FW}$ bits into a half-row buffer invalidates the contents of the other half-row buffer due to the sequential nature in which FW bits are read (as described in Section 3.1).

Dividing the row buffer into many shorter row buffers to achieve higher row buffer hit rate was previously proposed by Lee et al. [2009]. The main trade-off regarding the number of shorter row buffers to form is between benefiting from the multiple, separately managed buffers, while not being significantly deprived of exploiting spatial locality due to the shorter individual buffer length. SRB differs from this prior approach in that spatial locality is not affected (beyond how it is already affected by DBM). This is because the row buffer is divided between the FR and FW half-rows (that each have their own contiguous logical addresses). Thus, SRB provides a fine-granularity row buffer organization that can potentially improve MLC PCM performance and energy efficiency.

\section{IMPLEMENTATION AND COST}

We next discuss the changes in the PCM array, row buffer, memory controller, and processor to support DBM, APM, and SRB. 


\subsection{PCM Array}

DBM does not require any additional die area in the cell array, and can be implemented with small modifications to the control signal and bus organization. As discussed in Section 2.1, memory devices are accessed in two steps: first, the row decoder activates a row and its contents are stored in the row buffer; second, the column decoder selects a subset of the row buffer to be communicated with the I/O pads. With DBM, during row activation, an additional control signal decoded from the memory address (the region-select bit) notifies the sense amplifiers regarding whether to access the FR bits or FW bits. The overhead of this is small since a single signal can be shared by an entire row of sense amplifiers.

\subsection{Row Buffer}

To select a data block (cache block) in the row buffer, DBM requires reorganizing the latch-to-I/O multiplexers such that only FR bits or only FW bits are selected for communication with the I/O pads (the number of bits communicated is the same in $\mathrm{DBM}$ and the conventional scheme). Depending on the implementation of the sensing circuit, SRB will require reconfiguring the bit-line-to-latch multiplexers, such that FR bits and FW bits can be buffered in either of the two latches in the row buffer. These multiplexers are shown shaded in gray in Figure 8 and operate in parallel with the row decoder logic, which hides their latency.

We used NVSim [Dong et al. 2012] to calculate the cost of the additional multiplexing in a PCM memory modeled after that by Kang et al. [2007], and found the overall area of a 256MB MLC PCM array increases by $0.7 \%$ with SRB.

\subsection{Memory Controller}

The memory scheduler should be aware of the half-row access latencies when making scheduling decisions (these can be determined from the region-select bit) and the different half-row buffers within the row buffer. For SRB, an additional set of row buffer tags need to be maintained in the memory controller for determining what accesses can be serviced from either of the half-row buffers. For our system configuration, this requires an additional storage overhead of $40 \mathrm{~B}$.

\subsection{Processor}

APM requires maintaining a PC table of 16 program counter values and memory-write counters attributed to each. Assuming a 64-bit address space and 18-bit write counters, this amounts to $164 \mathrm{~B}$ of storage, which is $0.5 \%$ of the $\mathrm{L} 1$ cache size in our evaluated system. Augmenting the cache with a 4-bit field for each cache block increases the cache size by $0.7 \%$ for the both the L 1 and L 2 caches.

\section{EXPERIMENTAL METHODOLOGY}

For the evaluation of our proposed MLC PCM architecture, we developed a cyclelevel MLC memory timing simulator, which interfaces with an in-house x86 multicore simulator that has a front-end based on Pin. Representative phases of benchmarks were executed as profiled by PinPoints [Patil et al. 2004]. The major system parameters used in our study are shown in Table II. Results were collected for one billion cycles following a warmup period of 400 million cycles.

The use of a DRAM cache has been proposed in prior work to mitigate the latency, dynamic energy, and wearout issues of PCM [Qureshi et al. 2009b; Meza et al. 2012b; Dhiman et al. 2009]. In this study, we assume an on-chip eDRAM L3 cache similar to that of the IBM POWER7 processor [Kalla et al. 2010]. 
Table II. Major Simulation Parameters

\begin{tabular}{|c|c|c|c|c|}
\hline Processor & \multicolumn{4}{|c|}{8 cores, $4 \mathrm{GHz}$, single-issue in-order. } \\
\hline L1 cache & \multicolumn{4}{|c|}{ Private $32 \mathrm{~KB}$ per core, 4 -way, $64 \mathrm{~B}$ blocks, 3 cycle access latency. } \\
\hline L2 cache & \multicolumn{4}{|c|}{ Private $512 \mathrm{~KB}$ per core, 8-way, $64 \mathrm{~B}$ blocks, 12 cycle access latency. } \\
\hline L3 eDRAM cache & \multicolumn{4}{|c|}{ Shared $16 \mathrm{MB}, 16$-way, on-chip [kalla et al. 2010], 120 cycle access latency. } \\
\hline Memory controller & \multicolumn{4}{|c|}{$\begin{array}{l}2 \text { controllers each with } 64 \text {-bit channel, 128-entry read/write request queues, } \\
\text { FR-FCFS request scheduling [Rixner et al. 2000; Zuravleff and Robinson } \\
\text { 1997]. }\end{array}$} \\
\hline Main memory & \multicolumn{4}{|c|}{$\begin{array}{l}16 \text { GB MLC PCM, } 2 \text { ranks with } 8 \text { banks each, } 4 \mathrm{~K} \text { cells in a row ( } \times 8 \text { devices in } \\
\text { a rank). }\end{array}$} \\
\hline \multirow{5}{*}{ Memory timing } & & Baseline & FW bit & FR bit \\
\hline & Row buffer read & $12.5 \mathrm{~ns}$ & $12.5 \mathrm{~ns}$ & $12.5 \mathrm{~ns}$ \\
\hline & Row buffer write & $12.5 \mathrm{~ns}$ & $12.5 \mathrm{~ns}$ & $12.5 \mathrm{~ns}$ \\
\hline & Array read & $250 \mathrm{~ns}$ & $250 \mathrm{~ns}$ & $125 \mathrm{~ns}$ \\
\hline & Array write & $2 \mu \mathrm{s}$ & $1.68 \mu \mathrm{s}$ & $2 \mu \mathrm{s}$ \\
\hline \multirow{5}{*}{ Memory energy } & & Baseline & FW bit & FR bit \\
\hline & Row buffer read & $0.93 \mathrm{pJ} / \mathrm{bit}$ & $0.93 \mathrm{pJ} / \mathrm{bit}$ & $0.93 \mathrm{pJ} / \mathrm{bit}$ \\
\hline & Row buffer write & $1.02 \mathrm{pJ} / \mathrm{bit}$ & $1.02 \mathrm{pJ} / \mathrm{bit}$ & $1.02 \mathrm{pJ} / \mathrm{bit}$ \\
\hline & Array read & $10.89 \mathrm{pJ} / \mathrm{bit}$ & $10.89 \mathrm{pJ} / \mathrm{bit}$ & $5.68 \mathrm{pJ} / \mathrm{bit}$ \\
\hline & Array write & $368 \mathrm{pJ} / \mathrm{bit}$ & $272 \mathrm{pJ} / \mathrm{bit}$ & $368 \mathrm{pJ} / \mathrm{bit}$ \\
\hline
\end{tabular}

Memory timing and energy are based on Joshi et al. [2011], Qureshi et al. [2010a], Lee et al. [2009, 2010b], Yoon et al. [2012], and Lee et al. [2010a]. Note that peripheral circuitry latency and energy are accounted for when accessing the row buffer, which occurs on every access.

Table III. 8-Core Multithreaded Workloads Used in Our Study

\begin{tabular}{|c|c|c|c|}
\hline & & PCM banl & utilization \\
\hline Workload & Benchmarks & for reads & for writes \\
\hline MT1 & ScalarPenta $\times 8$ & $24.9 \%$ & $4.8 \%$ \\
\hline MT2 & DataCube $\times 8$ & $28.1 \%$ & $1.0 \%$ \\
\hline MT3 & Conj.Grad. $\times 8$ & $15.2 \%$ & $0.1 \%$ \\
\hline MT4 & IntegerSort $\times 8$ & $25.3 \%$ & $15.1 \%$ \\
\hline MT5 & ScalarPenta, DataCube, Conj.Grad $\times 4$, IntegerSort $\times 2$ & $23.2 \%$ & $3.9 \%$ \\
\hline MT6 & ScalarPenta, IntegerSort $\times 4$, DataCube $\times 3$ & $26.3 \%$ & $7.4 \%$ \\
\hline MT7 & IntegerSort $\times 5$, Conj.Grad, ScalarPenta, DataCube & $25.6 \%$ & $9.4 \%$ \\
\hline MT8 & ScalarPenta $\times 3$, IntegerSort $\times 3$, DataCube $\times 2$ & $26.3 \%$ & $5.4 \%$ \\
\hline
\end{tabular}

In order to examine the effectiveness of our memory system techniques, we examine a set of memory-intensive workloads. We use the four benchmarks from the NAS Parallel Benchmark suite and the six benchmarks from the SPEC CPU2006 suite that have misses per kilo-instructions (MPKI) higher than 1.75 and 2.5, respectively, at the on-chip eDRAM cache: Scalar Penta-diagonal solver, Data Cube, Conjugate Gradient, Integer Sort (Table III); and milc, mcf, omnetpp, libquantum, lbm, and soplex (Table IV). We form two groups of 8-core workloads from each benchmark suite: homogeneous workloads running multiple single-threaded instantiations of each benchmark and heterogeneous workloads formed by a random selection of benchmarks. We show the average PCM bank utilization for reads and writes for each of the workloads: the average fraction of cycles PCM banks spend servicing read and write requests. In addition, we evaluate the execution of 8 threads during the dominant parallel execution phase of the GraphLab framework for a Twitter social network data set [Kwak et al. 2010] (in Table V).

We adopt redundant bit-write removal in PCM to reduce memory wear. This involves writing to a cell only if the write data is different from that already stored in the cell. This technique removes $77 \%$ and $85 \%$ of writes in the baseline case and the DBM case, 
Table IV. 8-core Multiprogrammed Workloads Used in Our Study

\begin{tabular}{llcc}
\hline \multirow{2}{*}{ Workload } & \multicolumn{1}{c}{ Benchmarks } & \multicolumn{2}{c}{ PCM bank utilization } \\
for reads & for writes \\
\hline MP1 & milc $\times 8$ & $27.2 \%$ & $9.0 \%$ \\
MP2 & mcf $\times 8$ & $31.0 \%$ & $2.0 \%$ \\
MP3 & omnetpp $\times 8$ & $27.9 \%$ & $11.7 \%$ \\
MP4 & libquantum $\times 8$ & $12.4 \%$ & $0.001 \%$ \\
MP5 & lbm $\times 8$ & $22.8 \%$ & $24.7 \%$ \\
MP6 & soplex $\times 8$ & $27.3 \%$ & $7.2 \%$ \\
MP7 & omnet $\times 3$, milc, sop, mcf $\times 2$, libq & $27.8 \%$ & $6.8 \%$ \\
MP8 & milc $\times 2$, sop $\times 2$, lbm $\times 2$, mcf, omnet & $27.9 \%$ & $11.2 \%$ \\
MP9 & omnet $\times 2$, lbm, milc, sop $\times 2$, libq $\times 2$ & $26.8 \%$ & $8.6 \%$ \\
MP10 & milc, libq $\times 3$, lbm $\times 2$, omnet $\times 2$ & $26.2 \%$ & $10.2 \%$ \\
\hline
\end{tabular}

Table V. 8-Core Data-Intensive Parallel Workloads Used in Our Study

\begin{tabular}{llcr}
\hline \multirow{2}{*}{ Workload } & \multicolumn{1}{c}{ Benchmarks } & \multicolumn{2}{c}{ PCM bank utilization } \\
DI1 & GraphLab page_rank & $18.6 \%$ & $11.1 \%$ \\
DI2 & GraphLab connected_components & $31.8 \%$ & $8.0 \%$ \\
DI3 & GraphLab triangle_counting & $22.5 \%$ & $19.5 \%$ \\
\hline
\end{tabular}

respectively [Zhou et al. 2009; Jiang et al. 2012b]. Fewer writes are removed in the baseline case, as both the MSB and LSB of a cell have to match with the write data for the write to be redundant.

We report the speedup of multiprogrammed workloads using the weighted speedup metric [Snavely and Tullsen 2000], which is the sum of the speedups of the benchmarks when executed together on the evaluated system, relative to when executed individually on the baseline system. For parallel workloads, we report the speedup of the time it takes for all of the threads to complete execution. For multicore fairness, we use the maximum slowdown [Bender et al. 1998; Kim et al. 2010b, 2010c; Das et al. 2009; Vandierendonck and Seznec 2011] metric, which is the largest slowdown (reciprocal of speedup) experienced by any benchmark in the workload, compared to when that benchmark is run alone on the baseline system. Larger values of maximum slowdown indicate that some threads in the multicore workload are being unfairly slowed down compared to others, decreasing multicore fairness. The maximum slowdown metric is commonly used in the computer architecture community to evaluate multiprogrammed workload fairness.

\section{EXPERIMENTAL RESULTS}

We next discuss the impact of several different MLC PCM systems on system performance, energy efficiency, system fairness, and memory lifetime, as well as their sensitivity to different system configurations. For our study, we evaluated six MLC PCM systems:

-Baseline: The baseline MLC PCM architecture in which the bits of MLC cells are coupled together in the physical address space.

- All-FW: Models a scenario in which all of the pages are mapped to the FW region. Hence, memory writes always incur reduced latency and energy. Note that all of the pages fit within the 4GB of FW region memory available.

-All-FR: Models a scenario in which all of the pages are mapped to FR pages. In this case, memory reads always incur reduced latency and energy. Note that all of the pages fit within the $4 \mathrm{~GB}$ of FR region memory available. 


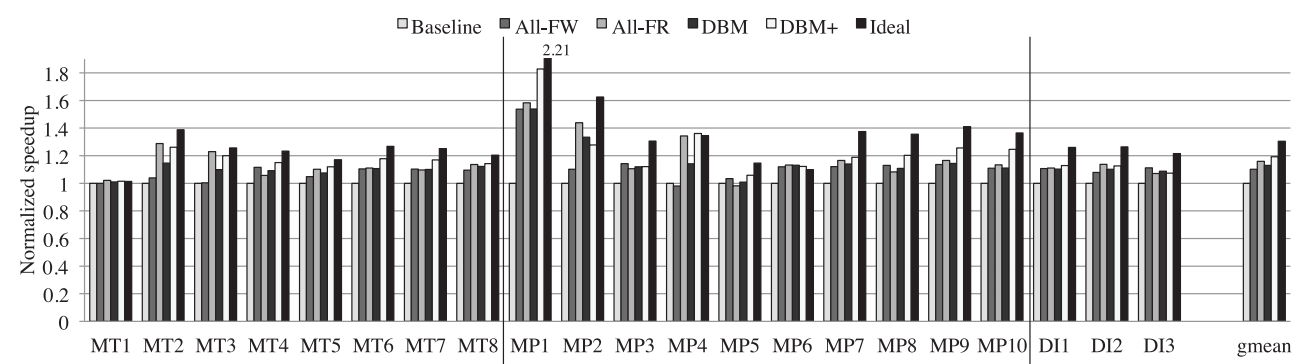

(a)

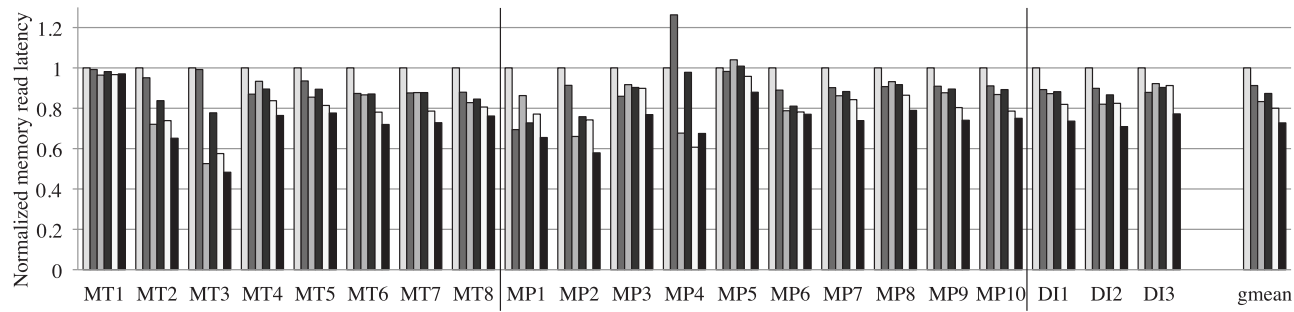

(b)

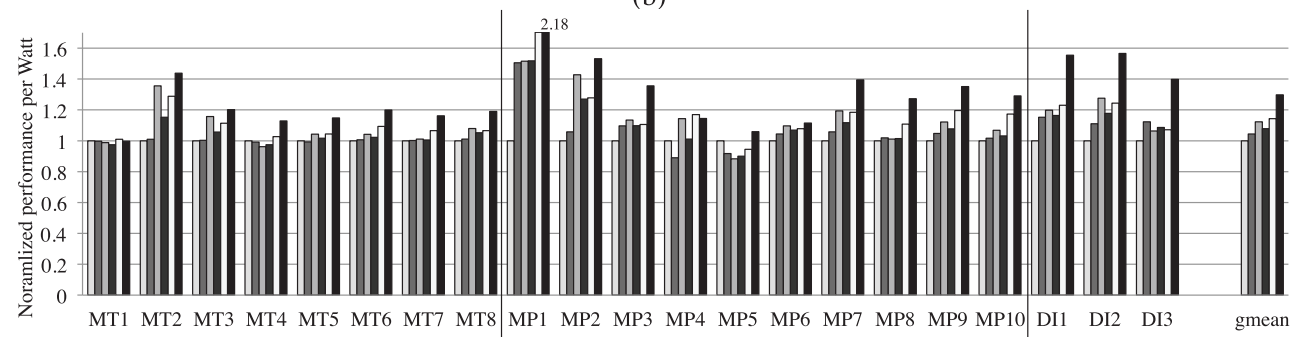

(c)

Fig. 9. Average (a) speedup, (b) memory read latency, and (c) energy efficiency.

-DBM: Our proposed decoupled bit mapping scheme that maps the FR bits and FW bits of cells to logically separate memory addresses.

$-D B M+A P M+S R B(D B M+)$ : In addition to DBM, this includes our proposed techniques that build on top of DBM: asymmetric page mapping and split row buffering.

-Ideal: This models an ideal scenario (in terms of cell access latency and energy) in which memory reads and writes are performed with the lowest latency and energy using a default mapping scheme.

\subsection{System Performance}

Figure 9(a) shows the speedup of each of our workload mixes for each of our evaluated systems normalized to the Baseline system. We draw three main observations from this data.

First, between the All-FW and All-FR systems, the All-FR system achieves higher performance (10.1\% better performance than the Baseline system for the All-FW system and $15.9 \%$ better performance than the Baseline system for the All-FR system). Interestingly, the benefits of the All-FR system come despite the fact that write latency is nearly an order of magnitude larger than read latency (Table II). This is due primarily to the read intensity of our workload mixes. This is illustrated for the MT workloads, for example, in the third column of Table I, which shows the fraction of pages that have at least a 10 to 1 read-to-write ratio. Figure 9(b) shows the average latency for memory 

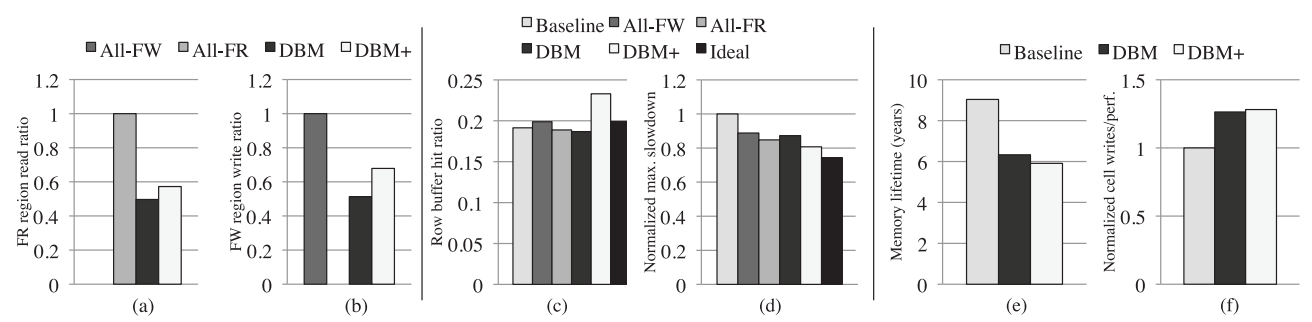

Fig. 10. Various metrics comparing the different MLC PCM systems. Ratio of (a) read accesses to FR region and (b) write accesses to FW region, showing that DBM+, by using APM, is able to make better use of the most efficient region for accessing data. (c) Row buffer hit ratio, showing that DBM+, by using SRB, is able to use better row buffer management to improve row buffer hit ratio. (d) Normalized max slowdown, an unfairness metric, which shows that most techniques improve multicore fairness compared to the baseline. (e) shows how the improved memory throughput and higher average number of bits written with DBM and DBM+ affect memory lifetime and (f) illustrates how DBM and DBM+ trade off memory endurance for additional performance.

read requests across the workloads for each of our evaluated systems. Notice that systems with lower average memory read latency have higher performance, confirming that system performance for our workloads running on an MLC PCM main memory is highly influenced by our workloads' memory read intensity.

Second, using DBM alone achieves performance in between the All-FW and All-FR systems (13.0\% higher performance than the Baseline system). This is because DBM provides both an FR region and an FW region, and the way an application's data is mapped to each region depends on its virtual-to-physical address mapping, which in our experiments has been randomized to simulate the effects of OS mapping. Thus, some of each application's data will be mapped into the FR region (with performance similar to the faster All-FR system) and some will be mapped into the FW region (with performance similar to the slower All-FW system).

Third, the DBM+ system, which incorporates our APM and SRB techniques, is able to achieve better performance than all the previously discussed systems $(19.2 \%$ better performance than the Baseline system) by more intelligently managing how data is stored and retrieved from main memory. We can see that it effectively helps bridge the gap between a DBM-only system and an unachievable Ideal system, which has $30.5 \%$ better performance than the Baseline system.

To help understand the results in greater detail, Figure 10(a) shows what fraction of read accesses are served in the FR region, and Figure 10(b) shows what fraction of write accesses are served in the FW region for the All-FW, All-FR, DBM, and DBM+ systems (note that for the Ideal system, the ratio would be 1.0 for both reads and writes). We can see that by employing APM, DBM+ is able to service a larger number of both reads and writes from the most efficient region of memory. In addition, Figure 10(c) shows what fraction of accesses are serviced from the row buffer at lower latency and lower energy than accessing the MLC PCM array. We can see that by employing SRB, DBM+ is able to achieve a higher row buffer hit ratio than the other techniques.

Some workloads do not follow these general patterns and are worth examining in more detail. MP1, MP9, and MP10 all benefit the most from the better page placement decisions of APM and additional amount of buffering provided by SRB in the DBM+ technique as opposed to simply using DBM alone. Note that the Ideal system only models ideal cell access latency and cell energy, not ideal data placement or data buffering, so that in some cases, such as MT1, MP4, and MP6 (which can perform better data placement and buffering), the Ideal latency and energy system does not perform the best. For MP4, the All-FR technique performs worse than the baseline; in 
MP5, the All-FW technique performs worse than the baseline because these workloads predominantly write data (in the case of MP4) and read data (in the case of MP5). This can be seen by the large improvement in performance for MP4 when using the All-FW technique and the improvement in performance for MP5 when using the All-FR technique (though the relative benefit for MP5 is less due to the lower latency of reads compared to writes). Finally, for workloads like MT4, MT7, MP3, MP5, MP8, and DI3, which perform many write accesses, the All-FW technique allows higher performance than the All-FR technique. For workloads like these, our assumption in APM that data should be allocated to the FR region by default can lead to lower performance than when always allocating data to the FW region (as it does with MP3 and DI3). In this case, system designers can choose to tailor the default data placement decisions of APM to their workloads' characteristics.

Overall, by employing our APM and SRB techniques on top of DBM, the DBM+ system is able to improve performance by $19.2 \%$ compared to the Baseline system and gets within $11 \%$ of the Ideal system.

\subsection{Memory Energy Efficiency}

The energy efficiency of the memory system is shown in Figure 9(c) in terms of system performance per memory Watt (higher is better). Compared to the Baseline, the All-FW system achieves energy benefits only on write accesses, which, as we have discussed, are not the predominant access type in our workloads, leading to only a small benefit in terms of energy-4.4\%. In contrast, the All-FR system improves energy efficiency by $12.2 \%$ compared to the Baseline system.

While the DBM system has the potential to get the best of both the All-FW and AllFR systems in terms of energy efficiency, because it uses a default mapping of memory and row buffer architecture, its energy efficiency lies in between the All-FW and AllFR systems, improving by only a modest $7.9 \%$ compared to the Baseline system. The $\mathrm{DBM}+$ system, on the other hand, is able to more efficiently utilize the FR and FW regions and more efficient row buffer organization provided by DBM, leading to an energy efficiency improvement of $14.4 \%$ compared to the Baseline system. In this way, the DBM+ system helps achieves energy efficiency within 16\% of the Ideal system.

\subsection{System Fairness}

Figure 10(d) shows the maximum slowdown (lower is better) of threads in the different MLC PCM systems. The improved performance of DBM contributes to lowering the maximum slowdown by $12.7 \%$ relative to the Baseline system. The DBM+ system further enhances thread fairness with (1) higher performance and (2) the SRB technique, which reduces the contention among threads for use of the row buffer, lowering the maximum slowdown by $19.3 \%$ relative to the Baseline system. This is better than the fairness of the All-FW and All-FR systems (11.2\% and $15.2 \%$ improvements, respectively, over the Baseline system), which also highlights the importance of optimizing for both reads and writes in PCM systems, which ASM is able to help toward doing.

\subsection{Memory Lifetime}

Figure 10(e) shows the projected operational memory lifetime in years. We assume a pessimistic cell endurance of $10^{6}$ writes and a wear-leveling scheme that distributes writes throughout 16GB of MLC PCM evenly. Two factors reduce the lifetimes of the $\mathrm{DBM}$ and DBM+ systems relative to the Baseline system. First, decoupled bit mapping doubles the number of cells that are associated with a block, since a block occupies one bit (FR or FW) from each cell. Thus, to write to a block, more cells potentially need to be programmed (however, the probability of redundant bit-writes is lower in the Baseline system than in DBM [Zhou et al. 2009; Jiang et al. 2012b]). Second, DBM and DBM+ 


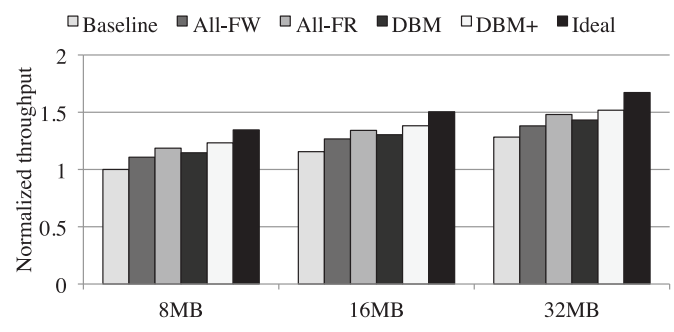

Fig. 11. Performance sensitivity to different last-level cache sizes.

have higher system performance, which causes the two systems to issue writes at a higher rate per unit time than the other systems, lowering memory lifetime.

To filter out the effect of system performance and isolate the effect of decoupled bit mapping on memory lifetime, we normalize the total number of cell-writes by performance in Figure 10(f). The overhead for the DBM and DBM+ systems in terms of normalized cell writes/performance are $26.4 \%$ and $28.2 \%$, respectively. We believe it is worthwhile to make this endurance tradeoff for performance, as recent work reports that much less pessimistic PCM endurance than we assumed for our study is possible ( $>10^{9}$ writes) [Kim et al. 2010a]. The idea is that the asymmetric read/write properties in MLC PCM devices will continue to exist (due to the multilevel sensing required for reads and the programming process required for writes), as PCM technology will improve in terms of endurance.

\subsection{Sensitivity to Last-Level Cache Size}

Figure 11 shows the sensitivity of performance (instruction throughput) to different last-level cache sizes (plots are normalized to the Baseline system with an 8MB lastlevel cache size). The trends for throughput improvement are similar for the different last-level cache sizes (as are the trends for speedup). Relative to the Baseline system in each individual category, DBM improves instruction throughput by $14.6 \%, 12.8 \%$, and $11.6 \%$ for the $8 \mathrm{MB}, 16 \mathrm{MB}$, and $32 \mathrm{MB}$ last-level cache sizes, respectively. For DBM+, the improvements are $23.3 \%, 19.6 \%$, and $18.3 \%$ for the same last-level cache sizes, outperforming the All-FR $(18.6 \%, 16.1 \%$, and 15.4\%) and All-FW (10.7\%, 9.6\%, and $7.7 \%$ ) systems. The Ideal system improvements are $34.5 \%, 30.2 \%$, and $30.3 \%$. With increasing last-level cache size, the performance improvement relative to the baseline decreases, as more requests for data are served by the last-level cache and less by the memory subsystem.

\section{RELATED WORK}

To our knowledge, this is the first work to (1) evaluate techniques to leverage MLC PCM's asymmetric access characteristics in the context of a PCM main memory, (2) propose a hardware/software data mapping technique to leverage the asymmetric access characteristics of MLC PCM, and (3) propose a low-overhead row buffer organization for MLC PCM that provides logically separate row buffers for each of the different bits in a multilevel cell. We next briefly discuss related research in other memory technologies, in MLC PCM devices, and in leveraging multiple row buffers in memory devices.

\subsection{Related Concepts in Other Memory Technologies}

The high-speed programming method in MLC Flash memory requires mapping the bits of an MLC cell to separate logical addresses. Although this resembles DBM, the two are fundamentally different. High-speed programming in Flash is designed to 
reduce the number of intermediate read verifications between successive programming iterations [Takeuchi et al. 1998]. On the other hand, DBM is motivated by MLC PCM's read and write asymmetry properties, which are exploited to expose the lower latency and energy of FR region reads and FW region writes. Jiang et al. [2012a] propose mapping the bits of an MLC STT-RAM cell to separate logical addresses to increase the performance of on-chip caches in embedded processors. Their mechanism migrates data to favorable memory locations depending on the observed type of accesses (reads or writes) frequently issued to the data. This is different from APM, which predicts whether data is read- or write-intensive before allocating it to memory locations, and our technique could potentially be used in STT-RAM caches. Recent work examining MLC resistive RAM [Niu et al. 2013] has looked at different ways of mapping data to cells to eliminate certain high-latency and high-energy states. These techniques could potentially be applied, in conjunction with our techniques, in MLC PCM cells that contain more than two levels (at only two levels, these techniques cannot leverage the capacity benefits of MLC).

\subsection{Multilevel Cell PCM}

Qureshi et al. [2010b] proposed dynamically adapting the usage of PCM either as high-density, high-latency MLC memory or as low-density, low-latency SLC memory, depending on the memory requirements of the application. Their work assumes that a system has overprovisioned memory capacity, and trades away surplus capacity for reduced latency. Our proposal reduces the latency while maintaining the high density of MLC PCM, through data mapping that exploits lower-bit latency and enables finegranularity row buffering. This does not prevent the memory from being configured as MLC or SLC, and is thus orthogonal work.

Other works have introduced ways to mitigate the high write latency of MLC PCM. Write cancellation and pausing allow reads to be served faster by interrupting long write operations [Qureshi et al. 2010a]. Jiang et al. [2012b] proposed write truncation that improves write latency by foregoing the programming of the few slower bits in a row (or block), compensating for the loss in data integrity with stronger ECC. They also proposed to store data in SLC form if the data and associated ECC bits can altogether be compressed to less than half of the original data size. These studies and others [Lee et al. 2010a; Yoon et al. 2012; Meza et al. 2012a] primarily focus on alleviating the high MLC PCM write latency (with read enhancements depending on the compressibility of the data). Both can be applied in conjunction with our proposals.

The practicality of MLC PCM technology has been demonstrated by various works [Bedeschi et al. 2009; Close et al. 2011; Papandreou et al. 2011]. Architectural solutions have been proposed to prolong memory lifetime through wear-leveling [Zhou et al. 2009; Qureshi et al. 2009a; Cho and Lee 2009], enhancing fault-tolerance [Ipek et al. 2010; Schechter et al. 2010; Yoon et al. 2011], data buffering [Qureshi et al. 2009b; Lee et al. 2009], and programming-current provisioning [Zhang et al. 2009]. These schemes are generic to SLC and MLC PCM, and can also be used in addition to our proposals.

\subsection{Multiple Row Buffers}

Several prior studies [Hsu and Smith 1993; Zhang et al. 2001; Loh 2008; Lee et al. 2009] evaluated the use of multiple row buffers in memory devices, showing that it generally leads to increased row buffer locality and memory performance gains. However, when resizing the length of each row buffer (e.g., for the purpose of reducing or maintaining the die area occupied by row buffers), Hsu and Smith [1993], Zhang et al. [2001], Lee et al. [2009], and Meza et al. [2012a] show that it harms performance if the row buffers are too short. This is because shorter row buffers exploit less spatial locality. In other words, for a constant die area dedicated to row buffers, there is a point at 
which increasing the number of row buffers at the expense of having them shorter is no longer desirable.

We propose a fine-granularity row buffer organization that divides the row buffer between the bits of an MLC cell (i.e., between the FR bits and the FW bits), instead of dividing the row buffer between the bitlines in a row. For DBM, where the bits of a cell are mapped to logically separate memory addresses, our scheme enables a fine-granularity row buffer organization while not diminishing spatial locality.

\section{CONCLUSION}

We presented new data mapping and buffering techniques that enable higher performance and energy efficiency by exploiting characteristics of multilevel cell phasechange memory technology. Our proposed approaches are built on decoupled bit mapping, which leverages the asymmetric read/write characteristics of MLC PCM cells. We examined two techniques enabled by decoupled bit mapping. Our first technique uses a hardware/software cooperative policy to map data in MLC PCM in a way that exploits the asymmetric read/write characteristics between the bits of an MLC PCM cell. The system software receives predictions from the hardware about the read/write affinity of data, then maps read-intensive pages to a fast-read/slow-write (FR) region and write-intensive pages to a slow-read/fast-write (FW) region. Our second technique exploits the flexibility in row buffering enabled by decoupled bit mapping to provide two logical row buffers from a single physical row buffer. This increases row buffer locality, enhancing both memory performance and energy efficiency. Our evaluations for a multicore system show that the proposed techniques provide better system performance, energy efficiency, and thread fairness compared to the conventional MLC PCM baseline. We believe that our proposals provide an effective way of taking advantage of MLC technology in main memory in a way that is also applicable to other emerging technologies with similar characteristics.

\section{ACKNOWLEDGMENTS}

We thank the anonymous reviewers and the members of the SAFARI research group for their comments and suggestions. This work is supported in part by NSF grants 0953246, 1212962, 1320531, the Intel Science and Technology Center for Cloud Computing, and the Semiconductor Research Corporation. We gratefully acknowledge the support of our industrial partners, including Hewlett-Packard Labs, IBM, Intel, Qualcomm, and VMware. Part of this work was done while HanBin Yoon and Justin Meza were interns at Hewlett-Packard Labs.

\section{REFERENCES}

L. A. Barroso and others. 2013. The Datacenter as a Computer. Morgan \& Claypool, San Francisco, CA.

F. Bedeschi and others. 2009. A Bipolar-Selected Phase Change Memory Featuring Multi-Level Cell Storage. JSSC (2009).

M. Bender and others. 1998. Flow and Stretch Metrics for Scheduling Continuous Job Streams. In SODA.

B. Buros. 2012. Tuning and Optimizing malloc on PowerLinux. IBM developerWorks (PowerLinux Architecture) (2012). Retrieved October 26, 2014 from http://www.ibm.com/developerworks/wikis/display/ LinuxP/Tuning+and+optimizing+malloc+on+PowerLinux.

Y. Cai and others. 2012. Error Patterns in MLC NAND Flash Memory: Measurement, Characterization, and Analysis. In DATE.

Y. Cai and others. 2013. Threshold Voltage Distribution in MLC NAND Flash Memory: Characterization, Analysis, and Modeling. In DATE.

S. Cho and H. Lee. 2009. Flip-N-Write: A Simple Deterministic Technique to Improve PRAM Write Performance, Energy and Endurance. In MICRO.

G. F. Close and others. 2011. A 512Mb Phase-Change Memory (PCM) in 90nm CMOS Achieving 2b/cell. In VLSIC.

R. Das and others. 2009. Application-Aware Prioritization Mechanisms for On-Chip Networks. In MICRO. 
G. Dhiman and others. 2009. PDRAM: a Hybrid PRAM and DRAM Main Memory System. In DAC.

W. Doerner. 2012. Memory Allocation and First-Touch. Intel Developer Zone (2012). http://software.intel.com/ en-us/articles/memory-allocation-and-first-touch.

X. Dong and others. 2012. NVSim: A Circuit-Level Performance, Energy, and Area Model for Emerging Nonvolatile Memory. TCAD (2012).

T. D. Happ and others. 2006. Novel One-Mask Self-Heating Pillar Phase Change Memory. In VLSIT.

M. Hosomi and others. 2005. A Novel Non-Volatile Memory with Spin Torque Transfer Magnetization Switching: Spin-RAM. In IEDM.

W.-C. Hsu and J. E. Smith. 1993. Performance of Cached DRAM Organizations in Vector Supercomputers. In ISCA.

E. Ipek and others. 2010. Dynamically Replicated Memory: Building Reliable Systems from Nanoscale Resistive Memories. In ASPLOS.

L. Jiang and others. 2012a. Constructing Large and Fast Multi-Level Cell STT-MRAM Based Cache for Embedded Processors. In DAC.

L. Jiang and others. 2012b. Improving Write Operations in MLC Phase Change Memory. In HPCA.

M. Joshi and others. 2011. Mercury: A Fast and Energy-Efficient Multi-Level Cell Based Phase Change Memory System. In HPCA.

R. Kalla and others. 2010. Power7: IBM's Next-Generation Server Processor. Micro, IEEE (2010).

P. Kaminski. 2009. NUMA Aware Heap Memory Manager. AMD Developer Central (2009). http://developer. amd.com/wordpress/media/2012/10/NUMA_aware_heap_memory_manager_article_final.pdf.

S. Kang and others. 2007. A 0.1-um 1.8-V 256-Mb Phase-Change Random Access Memory (PRAM) with 66-MHz Synchronous Burst-Read Operation. JSSC (2007).

I. S. Kim and others. 2010a. High Performance PRAM Cell Scalable to Sub-20nm Technology with below 4F2 Cell Size, Extendable to DRAM Applications. In VLSIT.

Y. Kim and others. 2010b. ATLAS: A Scalable and High-Performance Scheduling Algorithm for Multiple Memory Controllers. In HPCA.

Y. Kim and others. 2010c. Thread Cluster Memory Scheduling: Exploiting Differences in Memory Access Behavior. In MICRO.

H. Kwak and others. 2010. What is Twitter, a Social Network or a News Media? Retreived October 25, 2014 from http://an.kaist.ac.kr/traces/WWW2010.html. In WWW.

B. C. Lee and others. 2009. Architecting Phase Change Memory as a Scalable DRAM Alternative. In ISCA.

B. C. Lee and others. 2010a. Phase Change Memory and the Quest for Scalability. In CACM.

B. C. Lee and others. 2010b. Phase-Change Technology and the Future of Main Memory. In IEEE Micro.

G. H. Loh. 2008. 3D-Stacked Memory Architectures for Multi-core Processors. In ISCA.

J. Meza and others. 2012a. A Case for Small Row Buffers in Non-Volatile Main Memories. In ICCD.

J. Meza and others. 2012b. Enabling Efficient and Scalable Hybrid Memories Using Fine-Granularity DRAM Cache Management. CAL.

T. Nirschl and others. 2007. Write Strategies for 2 and 4-bit Multi-Level Phase-Change Memory. In IEDM.

D. Niu and others. 2013. Low Power Multi-Level-Cell Resistive Memory Design with Incomplete Data Mapping (ICCD).

N. Papandreou and others. 2011. Drift-Tolerant Multilevel Phase-Change Memory. In Intl. Memory Workshop $(I M W)$.

H. Patil and others. 2004. Pinpointing Representative Portions of Large Intel Itanium Programs with Dynamic Instrumentation. In MICRO.

M. K. Qureshi and others. 2009a. Enhancing Lifetime and Security of PCM-Based Main Memory with Start-Gap Wear Leveling. In MICRO.

M. K. Qureshi and others. 2009b. Scalable High Performance Main Memory System Using Phase-Change Memory Technology. In ISCA.

M. K. Qureshi and others. 2010a. Improving Read Performance of Phase Change Memories via Write Cancellation and Write Pausing. In HPCA.

M. K. Qureshi and others. 2010b. Morphable Memory System: a Robust Architecture for Exploiting MultiLevel Phase Change Memories. In ISCA.

M. K. Qureshi and others. 2012. PreSET: Improving Performance of Phase Change Memories by Exploiting Asymmetry in Write Times. In ISCA.

L. Ramos and others. 2011. Page Placement in Hybrid Memory Systems. In ICS.

S. Raoux and others. 2008. Phase-Change Random Access Memory: A Scalable Technology. IBM J. Res. Dev. 
S. Rixner and others. 2000. Memory Access Scheduling. In ISCA.

S. Schechter and others. 2010. Use ECP, Not ECC, for Hard Failures in Resistive Memories. In ISCA.

N. H. Seong and others. 2013. Tri-Level-Cell Phase Change Memory: Toward an Efficient and Reliable Memory System. In ISCA.

A. Snavely and D. M. Tullsen. 2000. Symbiotic Jobscheduling for a Simultaneous Multithreading Processor. In ASPLOS.

D. B. Strukov and others. 2008. The Missing Memristor Found. Nature.

K.-D. Suh and others. 1995. A 3.3 V 32 Mb NAND Flash Memory with Incremental Step Pulse Programming Scheme. In JSSC.

K. Takeuchi and others. 1998. A Multipage Cell Architecture for High-Speed Programming Multilevel NAND Flash Memories. JSSC (1998).

The International Technology Roadmap for Semiconductors. 2010. Process Integration, Devices, and Structures.

H. Vandierendonck and A. Seznec. 2011. Fairness Metrics for Multi-Threaded Processors. In CAL.

H. P. Wong and others. 2010. Phase Change Memory. In Proc. of the IEEE (2010).

D. H. Yoon and others. 2011. FREE-p: Protecting Non-Volatile Memory Against Both Hard and Soft Errors. In $H P C A$.

H. Yoon and others. 2012. Row Buffer Locality Aware Caching Policies for Hybrid Memories. In ICCD.

H. Yoon and others. 2013. Techniques for Data Mapping and Buffering to Exploit Asymmetry in Multi-Level Cell (Phase Change) Memory. Technical Report. Carnegie Mellon University, Pittsburgh, PA.

W. Zhang and others. 2009. Characterizing and Mitigating the Impact of Process Variations on Phase Change Based Memory Systems. In MICRO.

Z. Zhang and others. 2001. Cached DRAM for ILP Processor Memory Access Latency Reduction. In Micro, IEEE (2001).

P. Zhou and others. 2009. A Durable and Energy Efficient Main Memory Using Phase Change Memory Technology. In ISCA.

W. K. Zuravleff and T. Robinson. 1997. Controller for a Synchronous DRAM That Maximizes Throughput by Allowing Memory Requests and Commands to be Issued Out of Order. In U.S. Patent Number 5,630,096, Filed May 10, 1995, Issued May 13, 1997.

Received March 2014; revised June 2014; accepted September 2014 